\title{
Comparison of two VHIT software programs in diagnostics of semicircular canals
}

Piotr Pietkiewicz, Jaroslaw Milonski, Marzena Bielinska, Jurek Olszewski

Department of Otolaryngology, Laryngological Oncology, Audiology and Phoniatrics, Medical University of Lodz, Lodz, Poland

Submitted: 11 October 2016

Accepted: 14 November 2016

Arch Med Sci 2020; 16 (1): 124-128

DOI: https://doi.org/10.5114/aoms.2017.66048

Copyright $\odot 2017$ Termedia \& Banach

\section{Abstract}

Introduction: The aim of this work was to assess SCC function in VHIT test, using both versions of VHIT ULMER.

Material and methods: The study was performed in 57 subjects aged 22-33 years (48 women and 9 men) without vertigo in anamnesis. Patients did not complain of any "vertigo" ailments or any balance disorder incidents in the past. All tests using either VHIT I or VHIT II were performed by the same person. The function of lateral SCC in VHIT ULMER II was examined similarly like in VHIT ULMER I. In both methods was used the same sensitivity threshold parameters for horizontal and vertical accelerations (horizontal - 2500, vertical -1600). Each of these sequences enables a parameter, called represented GAIN, to be calculated in form of a point on the canalogram.

Results: GAIN represents the deviation of the gaze in comparison to the deviation of the head between the first $(t-0 \mathrm{~ms})$ and the fourth image $(t=120 \mathrm{~ms})$. The calculation formula is: GAIN (in \%) $=100 \times$ DG/RH. DG is deviation of the gaze ("gaze velocity in space") and $\mathrm{RH}$ is rotation of the head ("head velocity or impulse canal paresis"). Normal GAIN value ranges $1-40 \%$.

Conclusions: The examination using VHIT ULMER II appears to be a more sensitive diagnostic method than VHIT ULMER I. Improved software with automated functions such as camera adjustments, examination surface arrangement, of VHIT ULMER II, enables technician to shorten the time of examination, simultaneously providing a range of new information about the condition of oculo-vestibular system.

Key words: two VHIT software programs, semicircular canal diagnostics.

\section{Introduction}

Dizziness is a symptom reported by a large number of patients, and may be caused by a number of diseases or as an adverse effect of medications. Diagnosing the cause of the dizziness often requires the involvement of different health specialists. Head imaging techniques, such as computed tomography and magnetic resonance, are very important in the assessment of the causes of dizziness. In the vast majority of cases, after masses within the central nervous system have been excluded, tests to assess the balance system reactions are required. The currently applied tests for the balance system use physiological and non-physiological methods of stimulation, and advanced techniques measuring the responses to the stimuli. Video Head Impulse Test (VHIT) is a new diagnostic tool that, although still under development, has recently been

\author{
Corresponding author: \\ Prof. Jurek Olszewski \\ Department \\ of Otolaryngology, \\ Laryngological Oncology, \\ Audiology and Phoniatrics \\ Medical University of Lodz \\ 113 Zeromskiego St \\ 91-647 Lodz, Poland \\ Fax: +48 426393580 \\ E-mail: jurek.olszewski@ \\ umed.lodz.pl
}


made available for applications to evaluate the physiological and pathological processes of the balance system. The introduction of new diagnostic methods expands the diagnostic possibilities and allows for the more precise determination of the damaged place in the balance system. The VHIT is such a method, which tests oculovestibular reflexes for each of the three semicircular canals. The new version of VHIT extends the evaluation of the results [1-4].

The aim of this work was to assess the semicircular canals' function in the VHIT test, using both versions of VHIT ULMER.

\section{Material and methods}

The study was performed in 57 subjects aged 22-33 years (average: 24.9 years) -48 women and 9 men - without vertigo in their medical history. In the medical history, laryngological and otoneurological examinations and complete videonystagmography (VNG), no deviations in the vestibular organs were found in the studied patients.

All tests using either VHIT I or VHIT II were performed by the same person in all the patients.

The function of semicircular canals was examined in VHIT ULMER I in the following way: in a sitting position with the head leaned forward at a $30^{\circ}$ angle (Figure 1 ), the patient was looking at a motionless point while the examiner made rapid movements to the left and to the right (stimulation of the lateral semicircular canal, left or right). It was recorded by the VHIT-100 Ulmer Synapsis black-and-white video-camera with $1: 2.135 \mathrm{~mm}$ lens; 25 per second frame rate $-25 \mathrm{~Hz}$; x/y $72 \mathrm{dpi}$ resolution; 8-bit greyscale).

On examination of vertical semicircular canals the patient's head was leaned laterally towards the right side at a $45^{\circ}$ angle, then the examiner made repeated forward and backward movements, so the left anterior canal or right posterior canal was stimulated (Figure 2).

When the patient's head was leaned laterally towards the left side at a $45^{\circ}$ angle and after repeated forward and backward movements, the right anterior canal or the left posterior canal was stimulated.

Each of these sequences enables a parameter, called represented GAIN, to be calculated in the form of a point on the canalogram. The GAIN represents the deviation of the gaze in comparison to the deviation of the head between the first $(t-0 \mathrm{~ms})$ and the fourth image $(t=120 \mathrm{~ms})$. The calculation formula is as follows: GAIN (in \%) $=100$ $\times \mathrm{DG} / \mathrm{RH}$, where $\mathrm{DG}$ is the deviation of the gaze ("gaze velocity in space") and $\mathrm{RH}$ is the rotation of head ("head velocity or impulse canal paresis"). The normal GAIN value ranges from $1 \%$ to $40 \%$.

The function of lateral semicircular canals in VHIT ULMER II was examined similarly as in VHIT ULMER I. In both methods there were used the same sensitivity threshold parameters for horizontal and for vertical accelerations (horizontal acceleration - 2500, vertical acceleration - 1600).

The function of the canal was defined on the basis of the VOR amplification factor (the so-called VOR gain - gain of the vestibulo-ocular reflex).
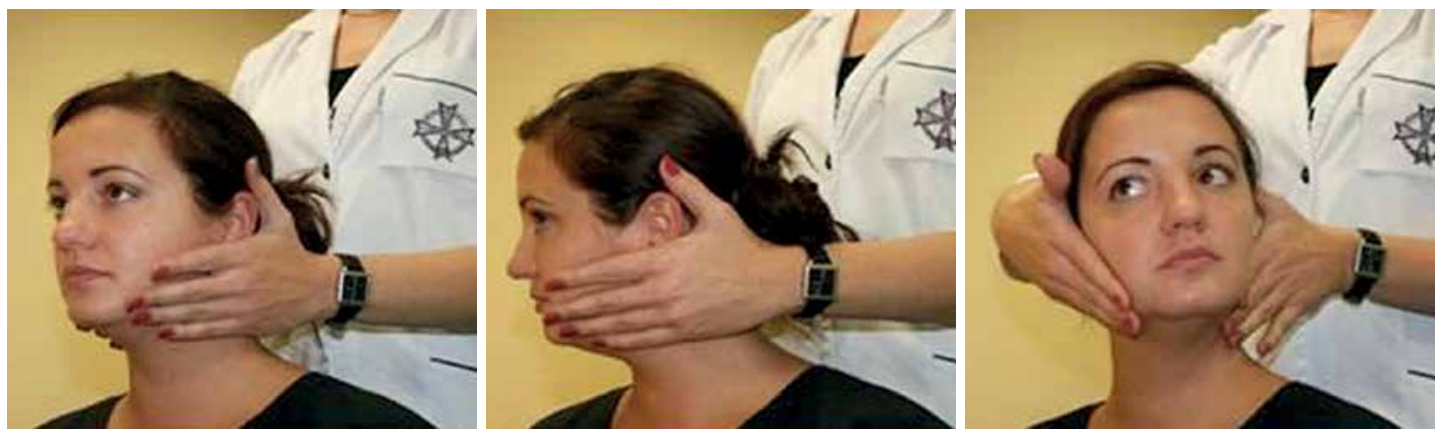

Figure 1. Range of head movements for testing the horizontal semicircular canals
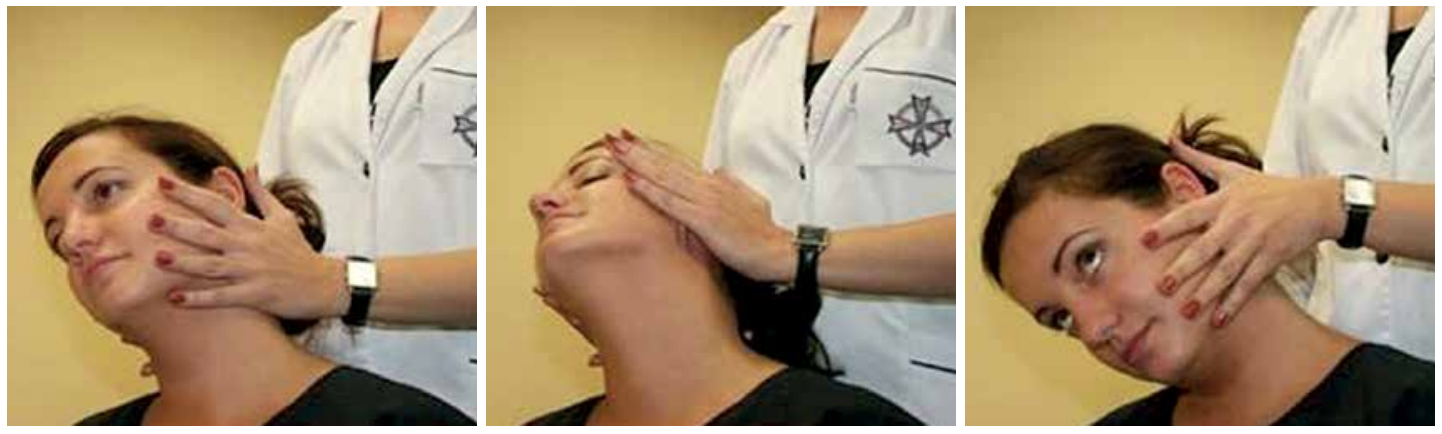

Figure 2. Range of head movements for testing the vertical semicircular canals 


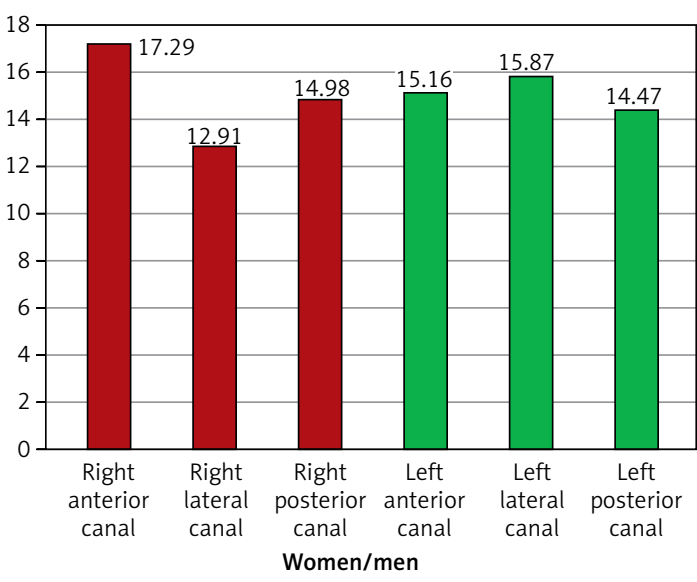

Figure 3. The mean value amplification/GAIN for VHIT ULMER I in women and men

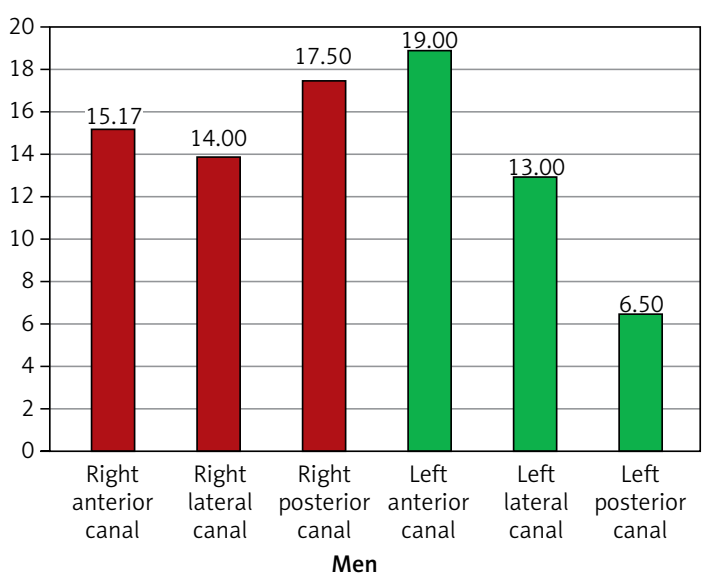

Figure 5. The mean value amplification/GAIN for VHIT ULMER I in men

The function of the canal was defined on the basis of GAIN, by the calculation formula as follows: VOR Gain = $1-\mathrm{DG} / \mathrm{RH}$, where DG stands for the deviation of gaze, $\mathrm{RH}$ stands for the rotation of head.

VHIT Ulmer II system parameters: field of vision (horizontal: $7.1^{\circ}$; vertical: $3.8^{\circ}$ ). Camera: cell type CCD 1/3", definition $752 \times 480$, frequency $100 \mathrm{~Hz}$. Infrared lighting: wavelength $830 \mathrm{~nm}$, power $70 \mathrm{~mW} / \mathrm{sr}$ per LED, light cone $\pm 22^{\circ}$, number of LEDs 56.

In the VHIT evolution the canal response is expressed in gain as an absolute. In the VHIT ULMER I, the canal response was expressed in deficit as

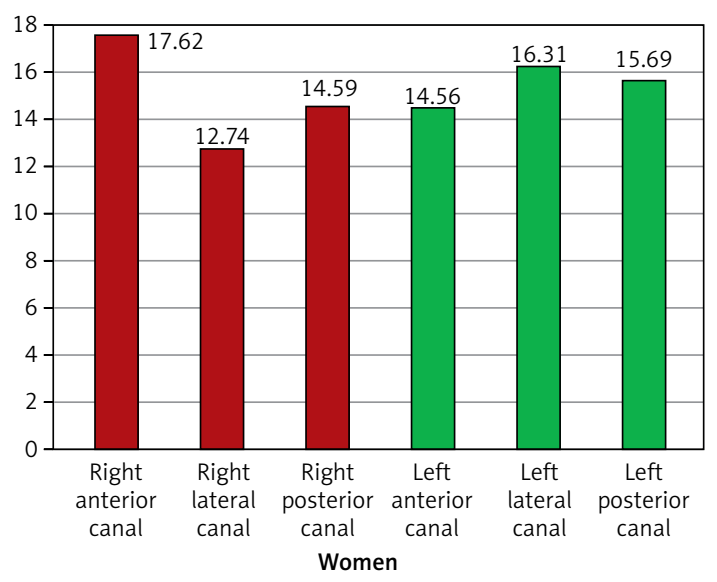

Figure 4. The mean value amplification/GAIN for VHIT ULMER I in women

a percentage. In the clinical papers, the gain as an absolute is more used, which is why we change the VHIT ULMER II for the absolute value.

If you want to compare both versions, you have to divide first the deficit (in \%) by 100 and then subtract it to: deficit (in percentage) $/ 100=$ deficit (in absolute), deficit (in absolute) = Gain (in absolute).

\section{Results}

In the test using VHIT ULMER I there were found no abnormal records within the scope of oculo-vestibular reflexes (Figures 3-5).

In the test using VHIT ULMER II the following abnormalities were discovered: 2 cases of damage of the right anterior semicircular canal, 1 case of damage of the left anterior semicircular canal, and 1 case of damage of the right lateral semicircular canal (Table I).

Average Ulmer II test values in men and women are presented in Figures 6-8.

\section{Discussion}

Despite highly specialist diagnostic methods, including videonystagmography and vestibular evoked myogenic potentials, the site of the injury is often hard to define precisely. It is particularly meaningful in the quick diagnostics for medical certification purposes.

A comparison between the latest model of the diagnostic device and its previous version appears

Table I. Amplification/GAIN for VHIT ULMER II in patients with incorrect records

\begin{tabular}{|c|c|c|c|c|c|c|}
\hline Patient & $\begin{array}{l}\text { Right anterior } \\
\text { canal }\end{array}$ & $\begin{array}{l}\text { Right posterior } \\
\text { canal }\end{array}$ & $\begin{array}{l}\text { Right lateral } \\
\text { canal }\end{array}$ & $\begin{array}{l}\text { Left anterior } \\
\text { canal }\end{array}$ & $\begin{array}{l}\text { Left posterior } \\
\text { canal }\end{array}$ & $\begin{array}{l}\text { Left lateral } \\
\text { canal }\end{array}$ \\
\hline 1 & 0.58 & & & & & \\
\hline 2 & & & 0.57 & & & \\
\hline 3 & & & & 0.3 & & \\
\hline 4 & 0.52 & & & & & \\
\hline
\end{tabular}




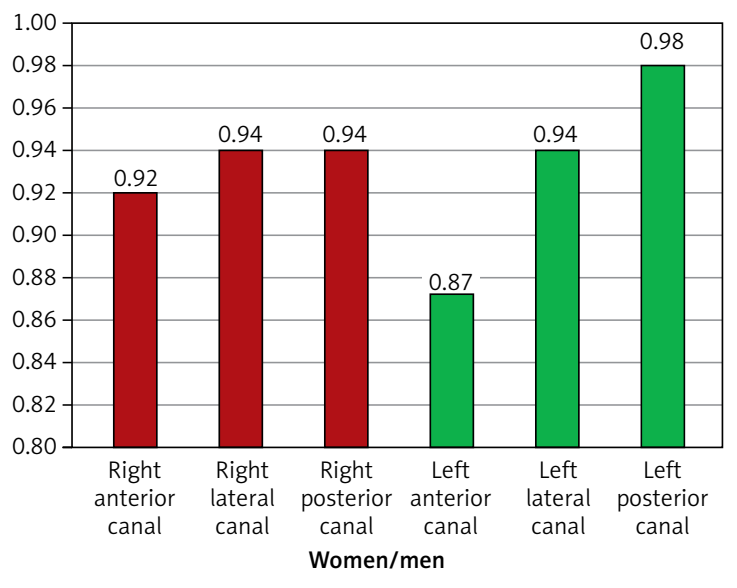

Figure 6. The mean value amplification/GAIN for VHIT ULMER II in women and men

to be the best method to analyse its sensitivity. To enhance the credibility of such comparative tests, a group of patients without any diseases or disorders of the ear, nose and throat (ENT) should be included.

In our study, we analysed a group of patients with no past medical history of dizziness or vertigo, although, as the study demonstrated, this did not always mean there was no damage within the balance system. Demonstrating this was not possible until a newer, more technologically advanced VHIT tool was employed. The VHIT Ulmer II was used to detect mild damage to the individual semicircular canals in 4 patients. These results were also important in the context of interpreting VHIT results that were not previously clear cut or unambiguous. Many authors have reported cases where patients with abnormalities found using sinusoidal tests gave normal VHIT results [5-7].

Therefore, many researchers considered this to be evidence that the two tests provided different kinds of information about the physiological and pathological processes in the balance system, and evaluated different elements. As we were in agreement with this opinion, we felt it important to note that the discrepancy might be associated with the insufficient sensitivity of the tools in detecting the parameters required for VHIT. The use of an ultra-sensitive camera combined with advanced, high-resolution software in the VHIT Ulmer II diagnostic tool enabled precise detection and analysis of eye movements in the plan view for head rotations during abrupt manoeuvres caused by the testing person [8-11].

Our investigation suggested that false-positive test results might be caused by the technological limitations. The use of a more advanced model of diagnostic tool should allow better correlations with nystagmography results and, as the only test of this type currently, provide information on the

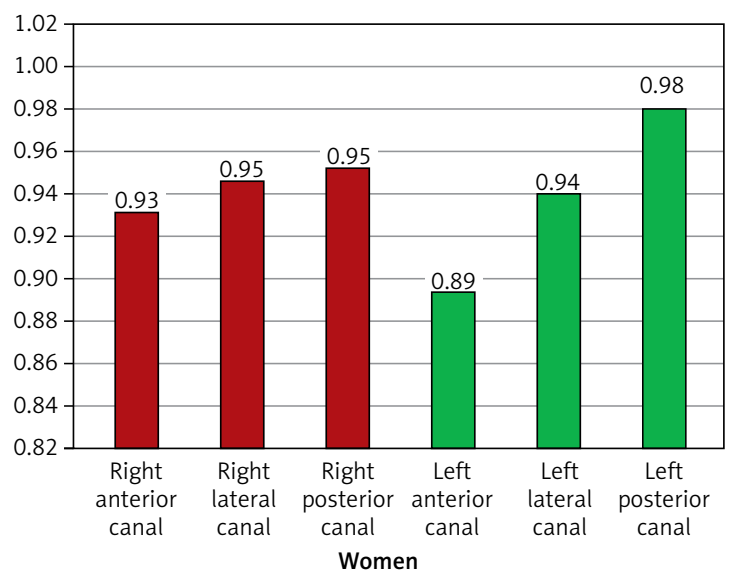

Figure 7. The mean value amplification/GAIN for VHIT ULMER II in women

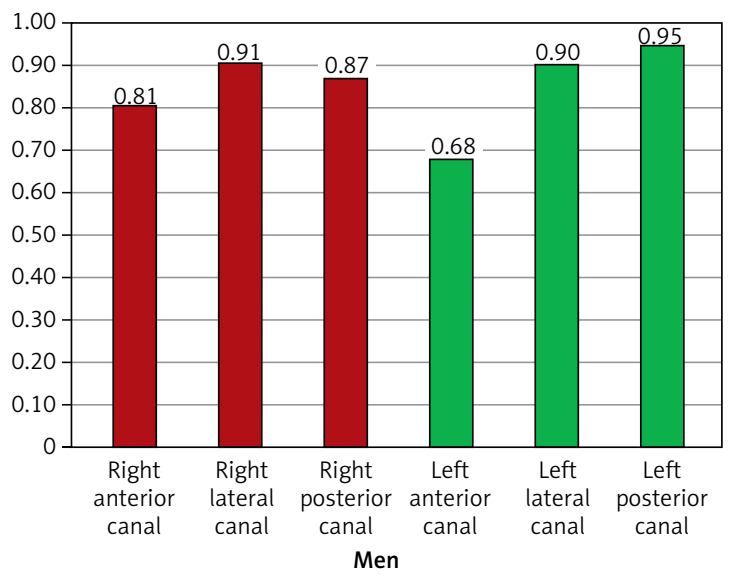

Figure 8. The mean value amplification/GAIN for VHIT ULMER II in men

functions of two other semicircular canals. Other advantages of the new VHIT model included reductions in the test time, allowing extended application of the method.

Like many other researchers, we realize that the practical application of VHIT is still associated with many limitations. Problems with precise head turning to stimulate the individual semicircular canals pose limitations on the use of VHIT. We still lack an appropriate device to precisely monitor the actual angle and velocity of head movements. The VHIT is a tool used to identify peripheral vestibular deficits, although unlike ENG or VNG it does not provide information about central damage to the balance system. In pursuing more sensitive diagnostic methods, we use newly developed technologies that enable precise detection of the analysed parameters [12-14].

The obtained data prove that VHIT allows for the definition of the site of the injury in the vestibular organ without any abnormalities in the full VNG test (including calibration, registration of possible spontaneous nystagmus, positional nystagmus, the alternate binaural bithermal caloric 
test with $\operatorname{cool} 30^{\circ} \mathrm{C}$ and warm $44^{\circ} \mathrm{C}$ air irrigations in the Fitzgerald-Hallpike test).

The aim of a recent study [15] was to assess the function of the semicircular canals in the videonystagmography head impulse test (VHIT ULMER I) in patients with vertigo and balance disorders diagnosed in the Department of Otolaryngology and Laryngological Oncology, Medical University of Lodz in whom there was identified vertigo of central, peripheral and mixed origins on the basis of complete videonystagmography.

The examination performed in 73 patients with vertigo of central, peripheral and mixed origins revealed semicircular canal injuries in 37 (50.69\%) patients, of whom 24 (32.87\%) patients showed an injury in one semicircular canal and $13(17.80 \%)$ patients in two or more canals.

Following the determination of the sensitivity of the VHIT ULMER II test in this study, a further study is planned using this test in vertigo patients in order to specify the site of the injury within the vestibular organ (semicircular canals) in quick diagnostics for medical certification purposes.

In conclusion, the examination using VHIT ULMER II appears to be a more sensitive diagnostic method than VHIT ULMER I. Improved software with automated functions, such as camera adjustments and examination surface arrangement, of VHIT ULMER II enables the technician to shorten the time of examination, simultaneously providing a range of new information about the condition of the oculo-vestibular system.

\section{Conflict of interest}

The authors declare no conflict of interest.

\section{References}

1. Zingler VC, Cnyrim C, Jahn K, et al. Causative factors and epidemiology of bilateral vestibulopathy in $255 \mathrm{pa}$ tients. Ann Neurol 2007; 61: 524-32.

2. McGarvie LA, Halmagyi M, Curthoys I, MacDougall $H$. Video head impulse testing - age dependent normative values in healthy subjects. J Vestib Res 2014; 24: 77.

3. Macdougall HG, McGarvie LA, Halmagyi GM, Curthoys IS, Weber KP. The video Head Impulse Test (VHIT) detects vertical semicircular canal dysfunction. PLoS One 2013; 8: 1488-98.

4. Curthoys IS. The interpretation of clinical tests of peripheral vestibular function. Laryngoscope 2012; 122: 1342-52.

5. Eza-Nuñez P, Fariñas-Alvarez C, Perez-Fernandez N. The caloric test and the Video Head-Impulse Test in patients with vertigo. Int Adv Otol 2014; 10: 144-9.

6. Bell SL, Barker F, Heselton H, Mackenzie E, Dewhurst D, Sanderson A. A study of the relationship between the video head impulse test and air calorics. Eur Arch Otorhinolaryngol 2015; 272: 1287-94.

7. Zulueta-Santos C, Lujan B, Manrique-Huarte R, PerezFernandez $\mathrm{N}$. The vestibulo-ocular reflex assessment in patients with Meniere's disease: examining all semicircular canals. Acta Otolaryngol 2014; 134: 1128-33.

8. MacDougall HG, Weber KP, McGarvie LA, Halmagyi GM, Curthoys IS. The video head impulse test: diagnostic accuracy in peripheral vestibulopathy. Neurology 2009; 73: 1134-41.

9. MacDougall HG, McGarvie LA, Halmagyi GM, Curthoys IS, Weber KP. Application of the video head impulse test to detect vertical semicircular canal dysfunction. Otol Neurotol 2013; 34: 974-9.

10. Weber KP, Aw ST, Todd MJ, et al. Inter-ocular differences of the horizontal vestibulo-ocular reflex during impulsive testing. Prog Brain Res 2008; 171: 195-8.

11. Matino-Soler E, Esteller-More E, Martin-Sanchez JC, Martinez-Sanchez JM, Perez-Fernandez N. Normative data on angular vestibulo-ocular responses in the yaw axis measured using the video head impulse test. Otol Neurotol 2015; 36: 466-71.

12. Mantokoudis G, Saber Tehrani AS, Kattah JC, et al. Quantifying the vestibulo-ocular reflex with video-oculography: nature and frequency of artifacts. Audiol Neurootol 2015; 20: 39-50.

13. McGarvie LA, Martinez-Lopez M, Burgess AM, MacDougall HG, Curthoys IS. Horizontal eye position affects measured vertical VOR gain on the video head impulse test. Front Neurol 2015; 6: 58-67.

14. Blödow A, Pannasch S, Walther LE. Detection of isolated covert saccades with the video head impulse test in peripheral vestibular disorders. Auris Nasus Larynx 2013; 40: 348-51.

15. Miłoński J, Pietkiewicz P, Bielińska M, Kuśmierczyk K, Olszewski J. The use of videonystagmography head impulse test (VHIT) in the diagnostics of semicircular canal injures. Int J Occup Med Environmen Health 2014; 27: 583-90. 